OPEN ACCESS

Edited by:

Lei Deng,

Central South University, China

Reviewed by:

Quan Zou,

University of Electronic Science and Technology of China, China

Yi Xiong,

Shanghai Jiao Tong University, China

${ }^{*}$ Correspondence: Weimin L

liweimin_2009@163.com

Specialty section:

This article was submitted to Statistical Genetics and Methodology, a section of the journal

Frontiers in Genetics

Received: 26 March 2021

Accepted: 12 April 2021

Published: 30 April 2021

Citation:

Yang W, Sun L, Cao X, Li L,

Zhang $X$, Li J, Zhao H, Zhan $C$, Zang Y, Li T, Zhang L, Liu G and Li W

(2021) Detection of circRNA

Biomarker for Acute Myocardial Infarction Based on System Biological

Analysis of RNA Expression.

Front. Genet. 12:686116.

doi: 10.3389/fgene.2021.686116

\section{Detection of circRNA Biomarker for Acute Myocardial Infarction Based on System Biological Analysis of RNA Expression}

\author{
Wen Yang ${ }^{1}$, Li Sun ${ }^{2}$, Xun Cao ${ }^{1}$, Luyifei Li' ${ }^{1}$ Xin Zhang', Jianqian Li', Hongyan Zhao ${ }^{3}$, \\ Chengchuang Zhan ${ }^{1}$, Yanxiang Zang ${ }^{1}$, Tiankai Li ${ }^{1}$, Li Zhang ${ }^{1}$, Guangzhong Liu' ${ }^{1}$ and \\ Weimin $L^{1 *}$

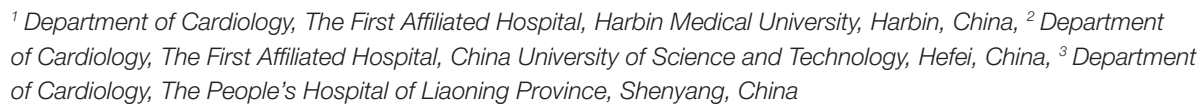

${ }^{1}$ Department of Cardiology, The First Affiliated Hospital, Harbin Medical University, Harbin, China, ${ }^{2}$ Department of Cardiology, The First Affiliated Hospital, China University of Science and Technology, Hefei, China, ${ }^{3}$ Department of Cardiology, The People's Hospital of Liaoning Province, Shenyang, China

Acute myocardial infarction (AMI) is myocardial necrosis caused by the persistent interruption of myocardial blood supply, which has high incidence rate and high mortality in middle-aged and elderly people in the worldwide. Biomarkers play an important role in the early diagnosis and treatment of AMI. Recently, more and more researches confirmed that circRNA may be a potential diagnostic biomarker and therapeutic target for cardiovascular diseases. In this paper, a series of biological analyses were performed to find new effective circRNA biomarkers for AMI. Firstly, the expression levels of circRNAs in blood samples of patients with AMI and those with mild coronary stenosis were compared to reveal circRNAs which were involved in AMI. Then, circRNAs which were significant expressed abnormally in the blood samples of patients with AMI were selected from those circRNAs. Next, a ceRNA network was constructed based on interactions of circRNA, miRNA and mRNA through biological analyses to detect crucial circRNA associated with AMI. Finally, one circRNA was selected as candidate biomarker for AMI. To validate effectivity and efficiency of the candidate biomarker, fluorescence in situ hybridization, hypoxia model of human cardiomyocytes, and knockdown and overexpression analyses were performed on candidate circRNA biomarker. In conclusion, experimental results demonstrated that the candidate circRNA was an effective biomarker for diagnosis and therapy of AMI.

Keywords: circRNA1, AMI 2, microarray 3, bioinformatics 4, circRNA_1047615

\section{INTRODUCTION}

AMI is myocardial necrosis induced by sudden occlusion of a coronary artery (Anderson and Morrow, 2017). In the past few decades, AMI has become a significant cause of emergency medical care, hospitalization, and death in China (Gao et al., 2008; Dai et al., 2017). Globally, the incidence of AMI is increasing year by year with a serious threat to human health and survival quality (Roger et al., 2012). Early diagnosis of AMI is critical for the appropriate initiation of life-saving treatment (Jeong et al., 2020). Biomarkers, such as creatine kinase isoenzyme (CKMB) 
and troponin I (TnI), are considered the gold standard for AMI. However, early diagnosis of AMI with borderline values of cardiac enzymes or waiting for serial changes could be challenging (Hajar, 2016). Therefore, a better understanding of the pathophysiological mechanisms of AMI and identifying new biomarkers for accurate and specific diagnosis are valuable.

Circular RNA (circRNA) is a type of single-stranded RNA that differs from well-known linear RNA by forming a covalently closed continuous loop (Zeng et al., 2017; Lu et al., 2019). They are generated by back-splicing of pre-mRNA transcripts, in which an upstream splice acceptor is connected to a downstream splice donor (Chen et al., 2019). The closed circular RNA was often considered a by-product of splicing error with little functional potential (Cocquerelle et al., 1993). However, based on the development of high-throughput sequencing, circRNAs have been found abundant, conserved, and specific, implying that they may possess biological and regulatory functions in the cytoplasm (Chen et al., 2019). Currently, circRNAs are found to have the following functions: they can modulate gene expression at the transcriptional or post-transcriptional level by sponging microRNAs (miRNAs) (Hansen et al., 2013; Wei et al., 2014); they can interact with RNA-binding proteins (Du et al., 2016; Wei et al., 2017); and they also have been shown to code for proteins (Begum et al., 2018). circRNA can serve as efficient miRNA sponges, interacting with miRNA to regulate mRNA expression. These specific functions and features of circRNAs suggest that they may be the ideal biomarkers to diagnose some human diseases rapidly.

Circular RNAs have been confirmed to be involved in the development of a variety of diseases (Zeng et al., 2020), including tumor system diseases (Xu et al., 2020), neurological disorders (Rybak-Wolf et al., 2015), endocrine system diseases (Gu et al., 2017), rheumatic system diseases (Luo et al., 2020), and cardiovascular diseases (CVD) (Geng et al., 2016; Wang et al., 2016) observed that circRNA HRCR acted as an endogenous miR-223 sponge to inhibit cardiac hypertrophy and heart failure. (Geng et al., 2016) found that over-expression of circRNA CDR1 in vivo increased the cardiac infarct size and suggested the potential of CDR1 was used as a new therapeutic target. These studies implied that circRNA may be a potential diagnostic biomarker and therapeutic target for CVD. However, few studies focused on the effect of circRNA on AMI. This study aimed to investigate the relationship between differentially expressed circRNA and AMI, and reveal the potential mechanisms via circRNA overexpression and knockdown. The ultimate goal was to provide new biomarkers for AMI diagnosis and new target for clinical treatment.

In this study, we performed a series of system biological analysis on RNA expression to find new effective circRNA biomarkers for AMI. The Arraystar Human Circular RNA Microarray Version 2.0 system was employed to detect the differential expression of circular RNAs in the whole blood of 8 patients ( 4 with acute myocardial infarction (AMI) and 4 with mild coronary artery stenosis). A total of 64 upregulated and 90 down-regulated circRNAs were identified using traditional statistical methods such as Student two-sample $t$ test and fold change. Therefore, five typical down-regulated
circRNAs were chosen for RT-qPCR validation. The relative expression levels of 3 circRNAs $(068655,104761$, and 104765) were consistent with the results of the microarray. TargetScan and miRanda databases were used to predict interactions between circRNAs and miRNAs. Furthermore, the circRNA-microRNAmRNA network was constructed. The prediction suggests that the circRNA_104761 can sponge microRNA-449 and microRNA34a, which are closely correlated with AMI. A larger scale sample experiment observed that the expression of circRNA_104761 was the highest in healthy volunteers, the second highest in mild coronary artery stenosis patients, and the lowest in AMI patients. The area under the receiver operating characteristic (ROC) curve for circRNA_104761 is 0.89 , implying a satisfactory prediction accuracy for AMI. To further verify the role of circRNA_104761 in AMI, the hypoxia model of human cardiomyocytes AC16 was established. All the experimental results demonstrated that circRNA_104761 could not only be an effective biomarker for AMI diagnosis, but also differentiate normal coronary artery, mild coronary artery stenosis, and AMI. Furthermore, circRNA_104761 may become a potential therapeutic target.

\section{MATERIALS AND METHODS}

\section{Overall Strategy}

Abnormally expressed circRNAs often affect the occurrence and development of diseases. To discover the circRNAs related to acute myocardial infarction (AMI), the expression levels of circRNA in blood samples of patients with AMI and those with mild coronary stenosis are firstly analyzed to find abnormally expressed (up-regulated or down-regulated) circRNAs. Next, differentially expressed circRNAs between AMI patients and mild coronary artery stenosis patients were analyzed with hierarchical clustering to find out the similarity of these whole blood samples. Then, RT-qPCR was performed to detect expression levels of circRNAs that were significantly abnormally expressed in blood samples of AMI patients. Afterward, TargetScan and miRanda databases were applied to obtain the data of circRNAsmiRNAs interaction to construct a ceRNA network involving three candidate circRNA biomarkers. Finally, according to the reported data of miRNA regulation of AMI, the circRNA involved in relevant regulation progression was identified and selected circRNA_104761 as candidate biomarker.

To determine the diagnostic potential of the circRNA biomarker selected by above methods for AMI, a series of biochemical experiments were performed. First of all, the expression levels of candidate circRNA biomarker in blood samples of AMI patients, mild coronary artery stenosis patients and normal coronary artery volunteers were detected. The expression levels of candidate circRNA were significantly different in these three groups, and it indicated that the circRNA biomarker was sensitive to AMI and can be used as diagnostic marker. Secondly, hypoxia is a direct consequence of AMI and an important factor leading to death. Subsequently, the expression levels of candidate circRNA biomarker in human cardiomyocytes under different hypoxia conditions were analyzed. The expression levels of candidate circRNA were 
significantly different under different hypoxia conditions, and it would suggest the circRNA biomarker can be used as molecular marker to determine the pathogenesis of AMI. Finally, the expression levels of candidate circRNA biomarker were intervened by either knockdown or overexpression, identified the influence on the occurrence and development of AMI. The overall strategy was illustrated in Figure 1A.

\section{Collection of Patient Samples and Ethics Statement}

Whole blood samples were collected from 34 AMI patients, 34 patients with mild coronary artery stenosis, and 30 volunteers with normal coronary arteries who attended the First Affiliated Hospital of Harbin Medical University (Harbin, China) in 2019. AMI patients were diagnosed based on acute ischaemictype chest pain, electrocardiogram (ECG), cardiac enzyme, and coronary angiography, etc. The patients with mild coronary artery stenosis and volunteers with normal coronary arteries were diagnosed by coronary CTA. Patients were excluded from malignant arrhythmia, cardiomyopathy, valvular heart disease, malignant tumors and rheumatic immune system diseases. Blood samples from the AMI patients were collected in $10 \mathrm{~min}$ when they arrived at the hospital before taking any medications. Patients with mild coronary artery stenosis and normal coronary artery volunteers were recruited at the time of the fasting blood in the morning. The clinical specimens were obtained from patients who gave informed consent. In the microarray experiment, blood samples from 4 AMI patients and 4 patients with mild coronary artery stenosis were collected. circRNA_104761 was further validated in 30 AMI patients, 30 patients with mild coronary artery stenosis, and 30 volunteers with normal coronary arteries using RT-qPCR. All patients were males, and their age was recorded. The clinical characteristics of the study populations are shown in Supplementary Table 1. The Harbin Medical University ethics committee approved all experimental protocols for the use of human samples, and the methods were carried out in accordance with the approved guidelines.

\section{Handing and Extraction Total RNA From Human Blood Samples}

Whole blood samples ( $1 \mathrm{~mL}$ per patient) were drawn from the study donors via direct venous puncture into $2.0 \mathrm{~mL}$ siliconized vacuum tubes containing $\mathrm{K} 2$ ethylene diamine tetraacetic acid (EDTA) for Microarray analysis and RT-qPCR. After blood collection, the blood samples were immediately placed into a liquid nitrogen tank and quickly transferred to an ultra-low temperature freezer at $-80^{\circ} \mathrm{C}$ for storage until use. This study extracted total RNA using TRI Reagent BD (Molecular Research Center, OH, United States).

\section{Microarray Hybridization and Data Analysis}

In this study, blood samples from 4 AMI patients and 4 patients with mild coronary artery stenosis were analyzed by the Arraystar Human circRNA Microarray version 2.0 system (Arraystar Inc, Rockville, MD, United States). Total RNA from each sample was quantified using the NanoDrop ND1000. All samples' preparation and microarray hybridization were conducted based on the Arraystar's standard protocols. Briefly, total RNAs were digested with Rnase R (Epicentre, Inc.) to remove linear RNAs and enrich circular RNAs. The enriched circular RNAs were then amplified and transcribed into fluorescent cRNA utilizing a random priming method (Arraystar Super RNA Labeling Kit; Arraystar, MD, United States). The labeled cRNAs were hybridized onto the Arraystar Human circRNA version 2.0 ( $8 \times 15 \mathrm{~K}$, Arraystar). After having washed the slides, the arrays were scanned by the Agilent Scanner G2505C. Agilent Feature Extraction software (version 11.0.1.1) was used to analyze acquired array images. Quantile normalization and subsequent data processing were performed using the $\mathrm{R}$ software. Before being used for the cluster analysis, the data were converted to standards. The function of dist and hclust were used to calculate distance and cluster, respectively. Hierarchical Clustering was performed to show the distinguishable expression profile of circRNAs between two groups. Differentially expressed circRNAs with statistical significance between the two groups were identified through Volcano Plot filtering. Differentially expressed circRNAs between two samples were identified through Fold Change filtering.

\section{RT-qPCR}

Total RNA was isolated from $1 \mathrm{~mL}$ whole blood using a phenolchloroform extraction procedure (Jiang et al., 2019; Liu et al., 2019), and RNA extraction process was performed as previously described (Section 2.2). circRNAs' relative expression level was detected by TB Green Premix Ex Taq II (TaKaRa Bio, Shiga, Japan) with $\beta$-actin as an internal control. The validation of all the circRNAs by qPCR was performed ViiA 7 Real-time PCR System (Applied Biosystems). $2^{-\Delta \Delta}$ Ct method was used to analyze the RT-qPCR data. Primers used in RT-qPCR for validation are shown in Supplementary Table 2.

\section{Cell Culture and Hypoxia Models}

Human myocardial cell AC16 was purchased from Shenzhen Haodi Huatuo Biotechnology Co Ltd. The basal medium was DMEM medium supplemented with $10 \%$ fetal bovine serum (Gibco) and $1 \%$ double antibody (100 U/ml penicillin and $100 \mu \mathrm{g} / \mathrm{ml}$ streptomycin, Invitrogen) at $37^{\circ} \mathrm{C}$ and $5 \% \mathrm{CO}_{2}$ atmosphere. For the hypoxia experiments, the cells were seeded in the anaerobic mode (oxygen concentration less than $0.1 \%$, carbon dioxide is $5 \%$, and the rest is nitrogen), and then used after 6,12 , and $24 \mathrm{~h}$ of treatment.

\section{Fluorescence in situ Hybridization (FISH)}

First, AC16 cell climbing slices were fixed in $4 \%$ paraformaldehyde (DEPC) for $20 \mathrm{~min}$, shaken, and washed 3 times with PBS ( $\mathrm{pH}$ 7.4) on a decolorizing shaker, and digested by dropping proteinase $\mathrm{K}(20 \mathrm{ug} / \mathrm{ml})$ for $8 \mathrm{~min}$. Then, pre-hybridization and hybridization were performed, blocking serum BSA, mouse anti-digoxigenin labeled peroxidase (antiDIG-HRP), and CY3-TSA was instilled in sequence, and stained with DAPI. Finally, we observed and collected images under fluorescence microscopy. 


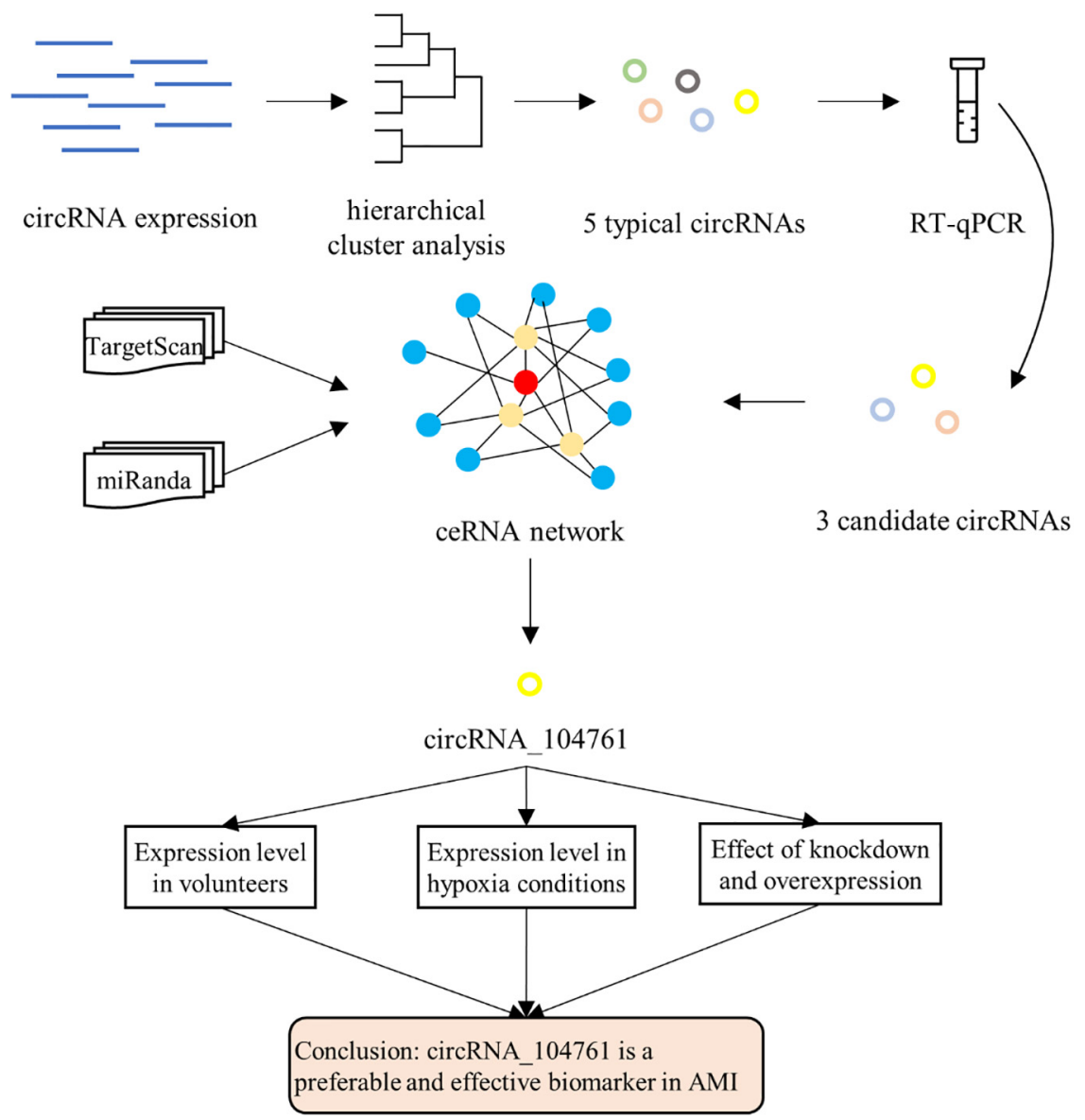

FIGURE 1 | Overall strategy.

\section{Knockdown and Overexpression of circRNA_ 104761}

The sequences of siRNA for circRNA_ 104761 used in this study were all synthesized by General Biological System (Anhui) Co Ltd, and three pairs of down-regulation primers were designed for circRNA_ 104761 at the same time. The sequences of primers are shown in Supplementary Table 3. The plasmid vector of circRNA_104761 overexpression model was also synthesized by General Biological System (Anhui) Co Ltd. Cell transfection was performed according to the manufacturer's instructions.

\section{CCK-8 Assay, LDH Assay, and Apoptosis Assay}

Cell Counting Kits (CCK-8 Kits) were purchased from Tongren Chemical (item number: CK04) to detect cell activity. LDH Assay Kits were purchased from Biyuntian Biotechnology Company (item number: C0017C0017). AnnexinV-FITC/PI Apoptosis Detection Kits were purchased from BD Company (item number: 556547). CCK-8 assay, LDH assay, and Apoptosis assay were all performed according to the kit's instructions.

\section{Statistical Analysis}

Statistical significance between groups was calculated by Student two-sample $t$-test. The diagnostic value of circRNAs was assessed by receiver operating characteristic (ROC) curves. SPSS statistics version 16.0 software (SPSS Inc, Chicago, IL, United States) was used to do the statistical analysis. A $p$-value $<0.05$ was considered to be significant. The Student two-sample $t$-test is expressed as:

$$
t(v(i))=\frac{m_{2}(i)-m_{1}(i)}{\sqrt{\frac{s_{1}^{2}(i)}{n_{1}}+\frac{s_{2}^{2}(i)}{n_{2}}}},
$$

where $i$ refers to the ith circRNA. $n_{1}$ and $n_{2}$ correspond to sample size of two groups. $m_{1}(i)$ and $m_{2}(i)$ represent the mean values of $i$ within the samples in each group. $s_{1}^{2}(i)$ and $s_{2}^{2}(i)$ denotes the corresponding sample variances. $v(i)$ refers to the freedom. That is:

$$
v(i)=\frac{\left(s_{1}^{2}(i) / n_{1}+s_{2}^{2}(i) / n_{2}\right)^{2}}{s_{1}^{4}(i) /\left[n_{1}^{2} \cdot\left(n_{1}-1\right)\right]+s_{2}^{4}(i) /\left[n_{2}^{2} \cdot\left(n_{2}-1\right)\right]} .
$$

In order to obtain ROC curves and the area under it (Wang et al., 2013; Zhao et al., 2015, 2017; Yang et al., 2020, 2021; Zhai et al., 2020), a certain classifier needs to be assigned. Here, we utilize 
Fisher's linear discriminative analysis. A direction vector $\boldsymbol{w}$ is to be determined, where data $\boldsymbol{x}$ is to be projected to obtain a value $y$. That is $y=\boldsymbol{w}^{t} \boldsymbol{x}$. The means of the two groups can be expressed as:

$$
\begin{aligned}
& \boldsymbol{m}_{\mathbf{1}}=\frac{1}{\mathrm{n}_{1}} \sum_{\mathrm{x} \in \mathrm{D}_{1}} \mathbf{x}, \boldsymbol{m}_{2}=\frac{1}{\mathrm{n}_{2}} \sum_{\mathrm{x} \in \mathrm{D}_{2}} \mathbf{x}, \mathrm{m}_{1}=\frac{1}{\mathrm{n}_{1}} \sum_{\mathrm{x} \in \mathrm{D}_{1}} \mathbf{w}^{\mathrm{t}} \mathbf{x} \\
& =\mathbf{w}^{t} m_{1}, m_{2}=\frac{1}{n_{2}} \sum_{x \in D_{2}} \boldsymbol{w}^{t} \mathbf{x}=w^{t} \boldsymbol{m}_{2},
\end{aligned}
$$

where $m_{1}, m_{2}, m_{1}$, and $m_{2}$ correspond to mean vectors and mean values of the two sample groups before and after projection. Also, we can get:

$$
\left|m_{1}-m_{2}\right|=\left|\boldsymbol{w}^{\mathrm{t}}\left(\boldsymbol{m}_{1}-\boldsymbol{m}_{2}\right)\right| .
$$

The covariance matrix of samples between classes is:

$$
S_{B}=\left(m_{1}-m_{2}\right)\left(m_{1}-m_{2}\right)^{t} .
$$

From equation (4) and equation (5), we can get:

$$
\begin{aligned}
& \left(m_{1}-m_{2}\right)^{2}=\left(\boldsymbol{w}^{t} \boldsymbol{m}_{1}-\boldsymbol{w}^{t} \boldsymbol{m}_{2}\right)^{2}=\boldsymbol{w}^{t}\left(\boldsymbol{m}_{1}-\boldsymbol{m}_{2}\right)\left(\boldsymbol{m}_{1}-\boldsymbol{m}_{2}\right)^{t} \boldsymbol{w} \\
& =w^{t} \boldsymbol{S}_{B} \boldsymbol{w} .
\end{aligned}
$$

Correspondingly, the covariance matrix of within class samples can be expressed as:

$$
\boldsymbol{S}_{w}=\sum_{x \in D_{1}}\left(\boldsymbol{x}-\boldsymbol{m}_{1}\right)\left(\boldsymbol{x}-\boldsymbol{m}_{1}\right)^{t}+\sum_{x \in D_{2}}\left(\boldsymbol{x}-\boldsymbol{m}_{2}\right)\left(\boldsymbol{x}-\boldsymbol{m}_{2}\right)^{t}
$$

From equation (7), we can get:

$$
\begin{aligned}
s_{1}^{2}+s_{2}^{2}= & \sum_{y \in D_{1}}\left(y-m_{1}\right)^{2}+\sum_{y \in D_{2}}\left(y-m_{2}\right)^{2} \\
= & \sum_{x \in D_{1}}\left(\boldsymbol{w}_{t} \mathrm{x}-\mathrm{w}_{t} \boldsymbol{m}_{1}\right)^{2}+\sum_{x \in D_{2}}\left(\boldsymbol{w}_{t} \mathrm{x}-\mathrm{w}_{t} \boldsymbol{m}_{2}\right)^{2} \\
= & \sum_{x \in D_{1}} \boldsymbol{w}^{t}\left(\boldsymbol{x}-\boldsymbol{m}_{1}\right)\left(\boldsymbol{x}-\boldsymbol{m}_{1}\right)^{t} \boldsymbol{w} \\
& +\sum_{x \in D_{2}} \boldsymbol{w}^{t}\left(\boldsymbol{x}-\boldsymbol{m}_{2}\right)\left(\boldsymbol{x}-\boldsymbol{m}_{2}\right)^{t} \boldsymbol{w}=\boldsymbol{w}^{t} \boldsymbol{S}_{w} \boldsymbol{w}
\end{aligned}
$$

From equation (6) and equation (8), the Optimization function is expressed as:

$$
J(\mathbf{w})=\frac{\left(m_{1}-m_{2}\right)^{2}}{s_{1}^{2}+s_{2}^{2}}=\frac{\boldsymbol{w}^{t} \boldsymbol{S}_{B} \boldsymbol{w}}{\boldsymbol{w}^{t} \boldsymbol{S}_{w} \boldsymbol{w}}
$$

Correspondingly, the best direction for projection can be obtained using following derivation. That is:

$$
\begin{aligned}
& \frac{\partial J}{\partial \boldsymbol{w}}=\frac{\left(\boldsymbol{S}_{B}+\boldsymbol{S}_{B}^{t}\right) \boldsymbol{w}}{\boldsymbol{w}^{t} \boldsymbol{S}_{w} \boldsymbol{w}}-\frac{\boldsymbol{w}^{t} \boldsymbol{S}_{B} \boldsymbol{w}\left[\left(\boldsymbol{S}_{w}+\boldsymbol{S}_{w}^{t}\right) \boldsymbol{w}\right]}{\left(\boldsymbol{w}^{t} \boldsymbol{S}_{w} \boldsymbol{w}\right)^{2}}=0 \Leftrightarrow \\
& \frac{2\left(\boldsymbol{w}^{t} \boldsymbol{S}_{w} \boldsymbol{w}\right) \boldsymbol{S}_{B} \boldsymbol{w}-2 \boldsymbol{w}^{t} \boldsymbol{S}_{B} \boldsymbol{w}\left(\boldsymbol{S}_{w}\right) \boldsymbol{w}}{\left(\boldsymbol{w}^{t} \boldsymbol{S}_{w} \boldsymbol{w}\right)^{2}}=0 \Leftrightarrow \\
& \boldsymbol{w}^{t} \boldsymbol{S}_{w} \boldsymbol{w} \boldsymbol{S}_{B} \boldsymbol{w}=\boldsymbol{w}^{t} \boldsymbol{S}_{B} \boldsymbol{w} \boldsymbol{S}_{w} \boldsymbol{w} \Leftrightarrow \\
& \lambda \boldsymbol{S}_{w}^{-1} \boldsymbol{S}_{B} \boldsymbol{w}=\boldsymbol{w}, \text { where } \boldsymbol{w}^{t} \boldsymbol{S}_{w} \boldsymbol{w} / \boldsymbol{w}^{t} \boldsymbol{S}_{B} \boldsymbol{w}=\lambda \Leftrightarrow \\
& \lambda \boldsymbol{S}_{\boldsymbol{w}}^{-1}\left(\boldsymbol{m}_{1}-\boldsymbol{m}_{2}\right)\left(\boldsymbol{m}_{1}-\boldsymbol{m}_{2}\right)^{t} \boldsymbol{w}=\boldsymbol{w} \Leftrightarrow \\
& \boldsymbol{w}=\lambda^{\prime} \boldsymbol{S}_{\boldsymbol{w}}^{-1}\left(\boldsymbol{m}_{1}-\boldsymbol{m}_{2}\right), \text { where } \lambda^{\prime}=\lambda\left(\boldsymbol{m}_{1}-\boldsymbol{m}_{2}\right)^{t} \boldsymbol{w}
\end{aligned}
$$

Regarding $\lambda^{\prime}$ as a scalar which can be omitted, the final direction vector $w$ can be expressed as:

$$
w=S_{w}^{-1}\left(m_{1}-m_{2}\right)
$$

Therefore, Fisher's linear discriminative analysis can be expressed as:

$$
\boldsymbol{w}^{t} \boldsymbol{x}+\boldsymbol{w}_{0}=\mathbf{0}
$$

where $\boldsymbol{w}_{0}=\boldsymbol{w}\left(\boldsymbol{m}_{1}+\boldsymbol{m}_{2}\right) / 2$.

\section{RESULTS}

\section{circRNA Expression Profiles of AMI and Mild Coronary Artery Stenosis Patients}

In this study, 64 up-regulated and 90 down-regulated circRNAs were identified in 4 AMI patients compared with 4 mild coronary artery stenosis patients (fold change $>2.0$ ) by microarray analysis (GSE169594), indicating these circRNAs were dysregulation. Differentially expressed circRNAs between AMI patients and mild coronary artery stenosis patients were subjected to hierarchical clustering analysis, suggesting the similarity of these whole blood samples. Hierarchical clustering revealed that the circRNA expression levels were distinguishable in the associated heat map (Figure 2A). A shorter distance generally indicates a high similarity. Therefore, Figure 2A shows that the circRNAs in the AMI patient group, and circRNAs in the mild coronary artery stenosis patient group had a relatively higher similarity. Box plot view (Figure 2B) shows the distribution of the hybridization data and degree of dispersion in AMI patients and mild coronary artery stenosis patients. The box plot shows that after $\log 2$ normalization, no abnormal distributions of data were observed in the 8 samples. The scatter and volcano plots shows varied circRNA expressions between the AMI and mild coronary artery stenosis samples (Figures 2C,D). In addition, a volcano plot identified differentially expressed circRNAs at different $p$-values and foldchanges between the two groups.

\section{In situ Validation of the Differentially Expressed circRNAs by RT-qPCR}

In terms of the microarray results, circRNAs were downregulated greater than up-regulate, so the down-regulated circRNAs were selected for continued validation. According to circRNA fold change values, $\mathrm{P}$ value magnitude, basic intensity of raw signal value (RawIntensity) (recommended above 200), number and sites of circRNA-bound miRNAs and current research status of bound miRNAs, five typical down-regulated circRNAs (hsa_circRNA068655, 089763, 103149, 104761, and 104765) were chosen (shown in Table 1) for further RTqPCR validation. As shown in Figure 3, the relative expression levels of 4 circRNAs (068655, 089763, 104761, and 104765) were down-regulated in $4 \mathrm{AMI}$ patients, which were consistent with the results of the microarray. However, the $p$-value of circRNA_089763 was over 0.05, which implied a nonsignificant expression difference of circRNA_089763 in two groups. In 
A

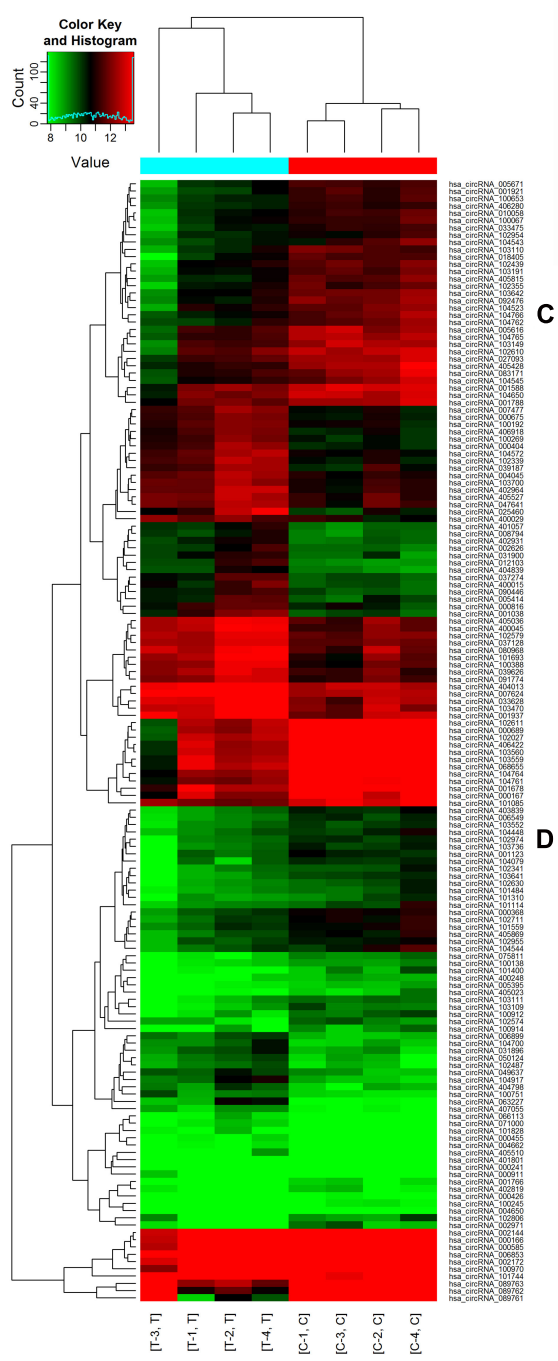

B

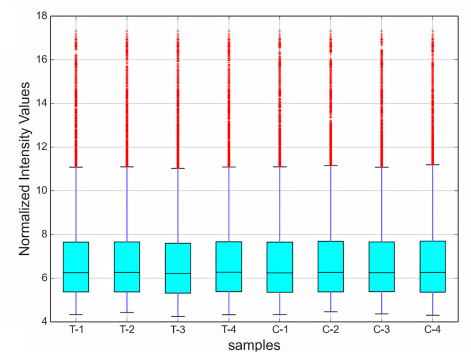

C

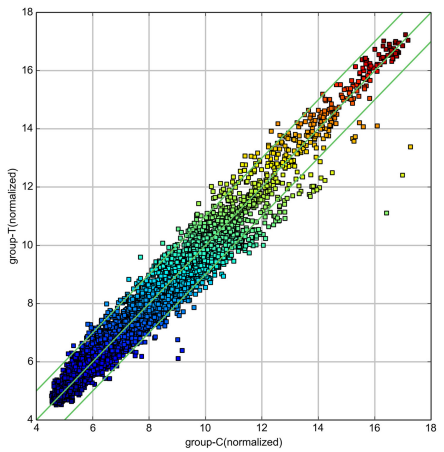

D

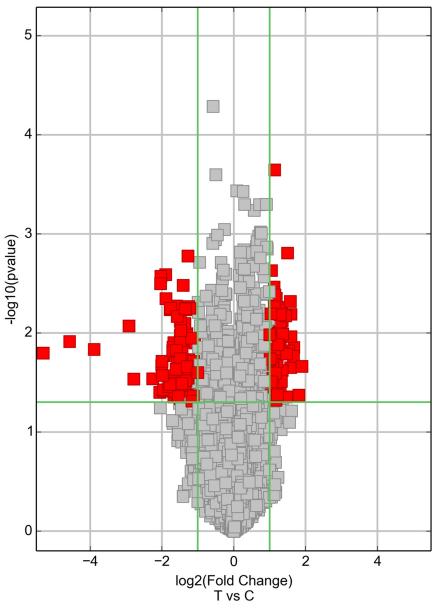

FIGURE 2 | circRNA expression profiles of AMI patients (Group T: T1, T2, T3, T4, and $n=4$ ) and mild coronary artery stenosis patients (Group C: C1, C2, C3, C4, and $n=4$ ) screened by microarray analysis. (A) Heat Map showing a distinguishable expression profile of circRNAs between two groups. Black stands for 0 , indicating no change in gene expression; red represents up-regulation, and green represents down-regulation. (B) Boxplot view showed the distribution of normalized expression intensity values for two groups. (C) Scatter plot indicated the variation of circRNA expression in AMl patients ( $y$-axis) and mild coronary artery stenosis patients ( $x$-axis). (D) Volcano plots visualizing differential circRNA expression between the two groups. The vertical lines correspond to a 2.0-fold change (FC) (log2 scaled) (up-regulation and down-regulation, respectively).

addition, the relative expression level of circRNA_103149 showed the opposite results of microarray analysis. Therefore, the circRNA_068655, circRNA_104761, and circRNA_104765 could be the potential biomarkers for AMI diagnosis and potential target for AMI treatment.

\section{Detailed Annotation for Interaction Between circRNA and miRNA}

This study applied TargetScan and miRanda to find the target miRNA which interacted with circRNAs (068655, 104761, and 104765) and predict the potential biological process in which the discovered circRNAs may participate in. The interaction between circRNAs (068655, 104761, and 104765) and corresponding miRNAs are shown in Table 2. circRNA _104761 may bind potential target miRNAs (hsa-miR-34c-5p, hsa-miRNA-449a, hsa-miRNA 449b-5p, hsa-miRNA-449c$3 p$, hsa-miR-370-3p) and the secondary structure of the binding site were predicted in Figure 4A. A circRNA-miRNAmRNA network was built by Cytoscape_3.7.0 as shown in Figure 4B. circRNA_104761 may sponge Hsa-miRNA-34a-5p, Hsa-miR-34b-5p, Hsa-miR-34c-5p, Hsa-miRNA-449a, 
TABLE 1 | Five typical down-regulated circRNAs in AMI patients identified by microarray analysis.

\begin{tabular}{|c|c|c|c|c|c|c|c|}
\hline circRNAs (has_circRNA) & Alias (has_circRNA) & Fold change & $P$-value & FDR & Regulation & circRNA type & Gene symbol \\
\hline 089763 & 0089763 & 14.83 & 0.014599771 & 0.684403672 & Down & exonic & JA760600 \\
\hline 104761 & 0001847 & 4.09 & 0.002673316 & 0.665381639 & Down & exonic & UBAP2 \\
\hline 068655 & 0068655 & 3.43 & 0.035551229 & 0.710909208 & Down & exonic & UBXN7 \\
\hline 104765 & 0001850 & 3.40 & 0.00561669 & 0.665381639 & Down & exonic & UBAP2 \\
\hline 103149 & 0002903 & 3.39 & 0.026855105 & 0.684403672 & Down & exonic & PCNT \\
\hline
\end{tabular}
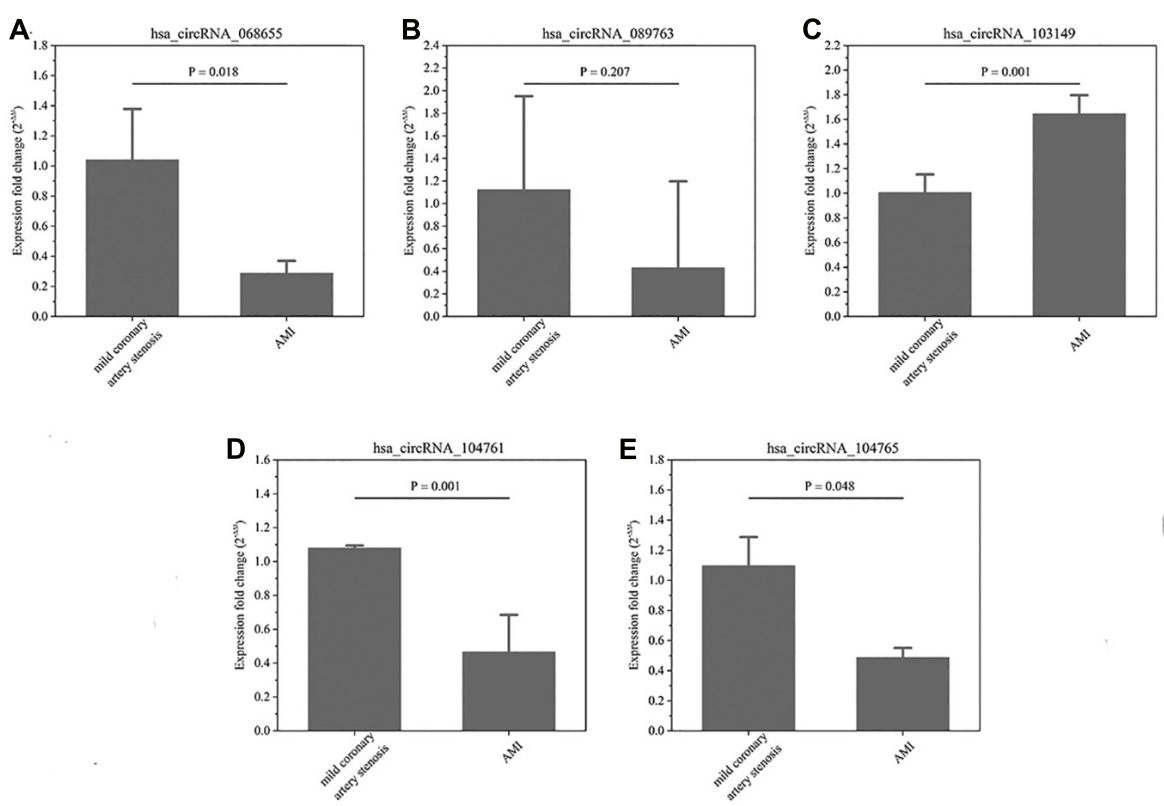

FIGURE 3 | (A-E) The relative expression of circRNAs. In-situ verification of the relative expression level of 5 down-regulated circRNAs (068655, 089763, 103149, 104761, and 104765) by RT-qPCR. Results are represented as means \pm standard deviation (SD). Data shown in the graphic was analyzed by independent sample $t$ tests with a significance level of $95 \%$.

Hsa-miRNA-449b-5p, and Hsa-miRNA-449c-3p. The circRNA_104761 can sponge microRNA-449 and microRNA-34a which is correlated with AMI (Fan et al., 2013; Zhang et al., 2019). Therefore, circRNA_104761 was selected for further validation in subsequent assays.

\section{Expression Levels of circRNA_104761 in the Whole Blood of 90 Volunteers}

The differential expressed circRNA_104761 was further validated in $30 \mathrm{AMI}$ patients, 30 mild coronary artery stenosis patients, and 30 normal coronary artery volunteers. As shown in Figure 5A, the average expression level of circRNA_104761 was significantly lower $(18 \%)$ in AMI patients $(0.639 \pm 0.217))$ than mild coronary artery stenosis patients $(0.824 \pm 0.216, p=0.002)$, suggesting that the predicated circRNA by microarray was effective in a larger scale sample. Besides, it is worthy to note that the expression difference of circRNA_104761 between mild coronary artery stenosis group $(0.824 \pm 0.216)$ and normal coronary artery group $(1.012 \pm 0.235)$ was also significant $(p=0.002)$, which implied the expression of circRNA_104761 in mild coronary artery stenosis patients had been inhibited.
The expression of circRNA_104761 was the highest in normal coronary artery volunteers, the second highest in mild coronary artery stenosis patients, and the lowest in AMI patients. The median cycle threshold (Ct) value for circRNA_104761 in 90 samples was 29.027, ranging from 27.623 to 32.971 . These results suggest that circRNA_104761 is sensitive and abundant in human blood.

\section{ROC Analysis of Validated circRNAs in AMI Patients and FISH}

The receiver operating characteristic (ROC) curves and the area under the ROC curve (AUC) were used to confirm the relationship between circRNA_104761 and AMI. As shown in Figure 5B, the AUC value of the ROC curve for circRNA_104761 was 0.890 (95\% confidence interval $[\mathrm{CI}]=0.807-0.973)$. Meanwhile, the sensitivity and specificity of the circRNA_104761 ROC curve were 0.867 and 0.800 , respectively. These results indicated that circRNA_104761 can be considered a preferable and effective biomarker for the diagnosis of AMI. RNA-FISH assay reveals that circRNA_104761 
TABLE 2 | TargetScan and miRanda predicted the interaction between circRNA and microRNA.

\begin{tabular}{lccccccc}
\hline circRNAs (has_circRNA) & T/C & $\boldsymbol{p}$-value & MRE1 & MRE2 & MRE3 & MRE4 \\
\hline 068655 & 0.27 & 0.00441 & hsa-miR-3140-3p & hsa-miR-4539 & hsa-miR-3660 & hsa-miR-4260 & hsa-miR-3118 \\
104761 & 0.43 & 0.03983 & hsa-miR-34c-5p & hsa-miR-449a & hsa-miR-449b-5p & hsa-miR-449c-5p & hsa-miR-370-3p \\
104765 & 0.44 & 0.04856 & hsa-miR-532-5p & hsa-miR-496 & hsa-miR-767-5p & hsa-miR-589-5p & hsa-miR-188-3p
\end{tabular}
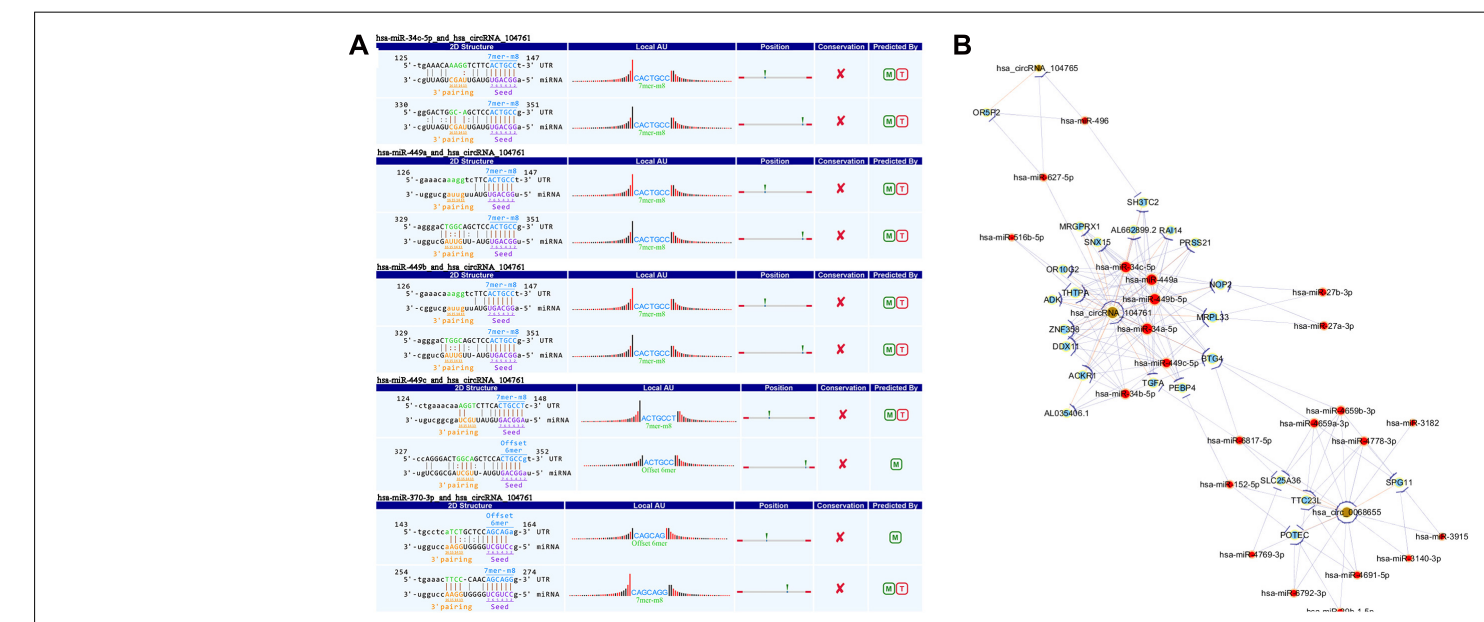

FIGURE 4 | The predicted miRNAs. (A) TargetScan and miRanda predicted the 5 most potential target miRNAs that may bind to circRNA_104761 and the binding site's secondary structure. (B) Network of circRNA $(068655,104761$, and 104765) -miRNA-mRNA.
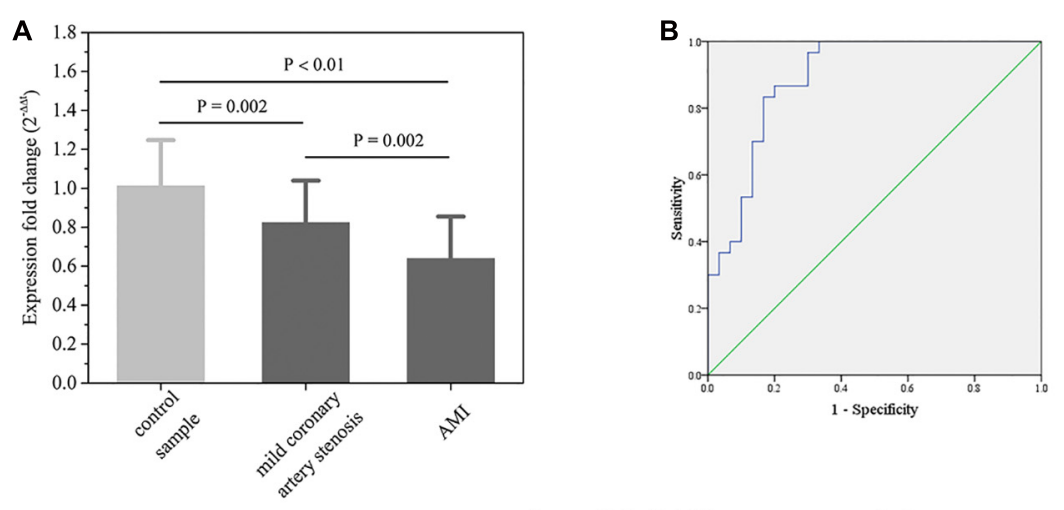

C

DAPI

circ_104761

Merge

AC16
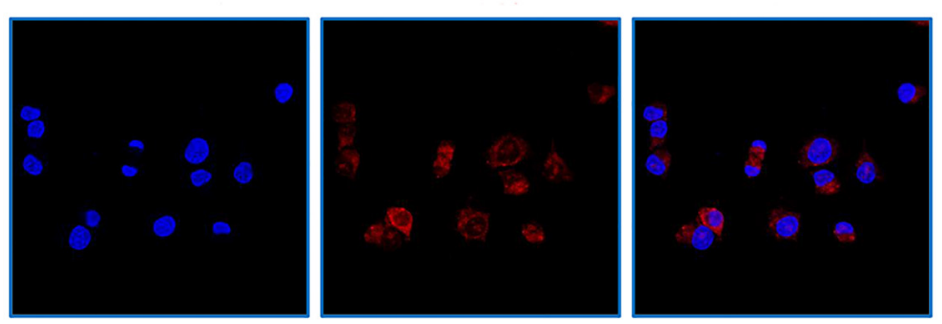

FIGURE 5 | The relative expression of circRNA_104761 in larger scale and the ROC curve analysis. (A) Verification of the relative expression level of down-regulated circRNA_104761 by RT-qPCR in expanded sample test. (B) ROC curve analysis evaluating the diagnostic value of validated circRNA_104761 for AMI.

(C) RNA-FISH assays determined the subcellular localization of circRNA_104761 in AC16. Results are represented as means \pm standard deviation (SD). Data shown in the graph was analyzed by independent sample $t$-tests with a significance level of $95 \%$. 

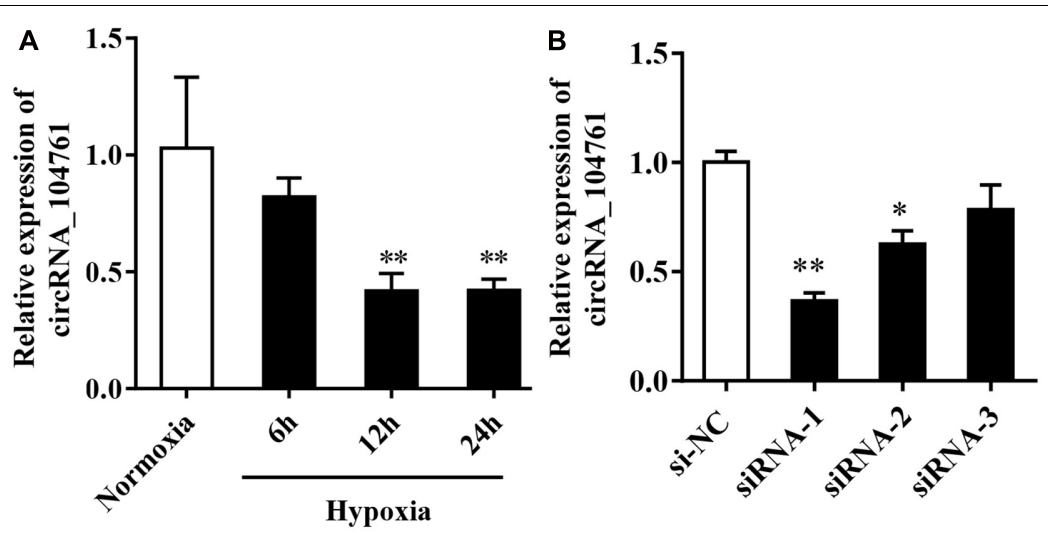

C
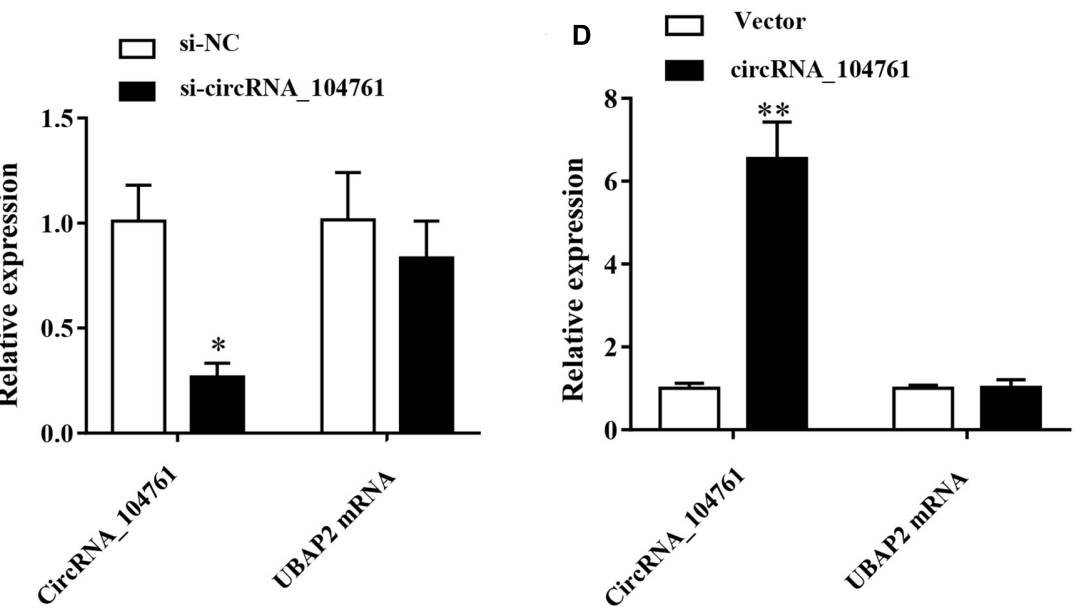

FIGURE 6 | Expression of circRNA_104761 in hypoxic human cardiomyocytes AC16 and the circRNA_104761 editing efficiency. (A) Expression of circRNA _ 104761 in AC16. (B) The down-regulation efficiency of three siRNAs (siRNA-1, siRNA-2, and siRNA-3). (C) The effect of siRNA-1 on endogenous circRNA_104761 and its source gene UBAP2. (D) The over-expression efficiencies of circRNA_104761 and UBAP2. ${ }^{*} p<0.05$ and ${ }^{* \star} p<0.01$.

(FAM-labeled) distinctly distributed in the cytoplasm of AC16 cells (Figure 5C).

\section{Expression of circRNA_104761 in Hypoxic Human Cardiomyocytes AC16 and the circRNA_104761 Editing Efficiency}

Hypoxia is an important factor causing myocardial injury. After cardiomyocytes AC16 were treated with hypoxia condition for $6 \mathrm{~h}$ (6 h group), $12 \mathrm{~h}$ (12 h group), and $24 \mathrm{~h}$ ( $24 \mathrm{~h}$ group), and the expression of circRNA_104761 was detected by RT-qPCR. The results showed that the expression level of circRNA_104761 in hypoxic cardiomyocytes AC16 (Hypoxia, 6, 12, and 24h) was inhibited compared with normal AC16 (Normoxia), and the expression level of circRNA_104761 in hypoxic cardiomyocytes AC16 (12h, 24h) was significantly decreased (Figure 6A, $p<0.01)$. Hypoxia treatment for $12 \mathrm{~h}$ was chosen for subsequent experiments. To further investigate the effect of circRNA_104761 on cardiomyocytes, we constructed siRNAs that could knockdown the expression of circRNA_104761. This study designed three pairs of siRNAs (siRNA-1, siRNA-2, and siRNA-3) to verify the down-regulation efficiency by RT-qPCR. The results showed that siRNA-1 and siRNA-2 down-regulation efficiency was significant (Figure 6B). Moreover, siRNA-1 was selected for further experiments. The source gene of circRNA_104761 was UBAP2 mRNA by circbase query, and specific siRNA-1 was able to significantly interfere with endogenous circRNA_104761, but had no significant effect on its source gene (UBAP2) (Figure 6C). Furthermore, we constructed a plasmid vector to overexpress circRNA_104761 in AC 16, and RT-qPCR was used to verify the overexpression efficiency. The results showed that the overexpression efficiency of the constructed plasmid vector was significant. The specific plasmid vector could significantly overexpress circRNA_104761, but had no significant effect on its source gene (UBAP2) (Figure 6D). Therefore, this plasmid vector was used for the following experiments.

\section{Effect of circRNA_104761 Knockdown and Overpression in Human Cardiomyocytes AC16}

After knockdown of circRNA_104761 with siRNA, LDH assay demonstrated LDH activity increased after hypoxia treatment, 

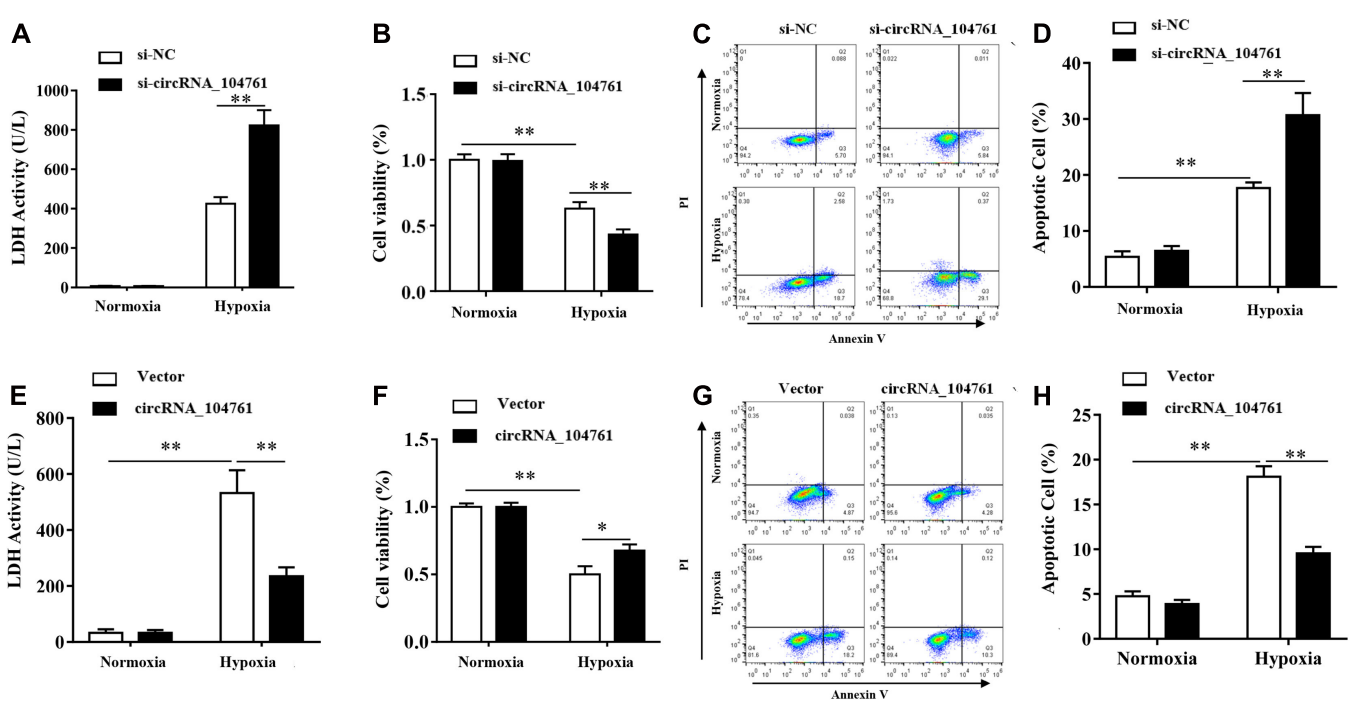

FIGURE 7 | Effect of circRNA_104761 knockdown and overpression in human cardiomyocytes AC16. (A-D) Effect of circRNA_104761 knockdown on AC16. (A) LDH activity test (Normoxia and Hypoxia). (B) Cell viability was determined by CCK8 assay. (C,D) Flow data analysis of apoptosis. (E-H) Effect of circRNA_104761 overexpression on AC16. (E) LDH activity test (Normoxia and Hypoxia). (F) Cell viability was determined by CCK8 assay. (G,H) Flow data analysis of apoptosis. Results are represented as means \pm standard deviation (SD). Data shown in this graph was analyzed by independent sample $t$-tests with a significance level of $95 \%$. ${ }^{* \star} p$-value $<0.01$ and ${ }^{*} p$-value $<0.05$.

and that down-regulation of circRNA_104761 significantly increased the release of lactate dehydrogenase in AC16 cell after hypoxia treatment (Figure 7A, $p$-value $<0.01$ ). CCK8 assay demonstrated cell viability was significantly lower after hypoxia treatment, and that down-regulation of circRNA_104761 significantly reduced AC16 cell viability after hypoxia condition (Figure 7B), which enhanced AC16 cell death. Flow cytometry data demonstrated apoptosis was significantly increased after hypoxia, and that down-regulation of circRNA_104761 significantly exacerbated the early apoptotic level of AC16 cells after hypoxia treatment (Figures 7C,D).

When the expression of circRNA_104761 was overexpressed by the constructed plasmid vector, LDH activity was significantly increased after hypoxia, and the results of $\mathrm{LDH}$ tests demonstrated that exogenous overexpression of circRNA_104761 significantly decreased the release of lactate dehydrogenase in AC16 cell after hypoxia treatment (Figure 7E, $p<0.01$ ). CCK8 assay demonstrated cell viability was significantly lower after hypoxia treatment, and that the overexpression of circRNA_104761 significantly increased AC16 cell viability after hypoxia treatment (Figure 7F). Flow cytometry data demonstrated apoptosis significantly increased after hypoxia treatment, and that overexpression of circRNA_104761 significantly alleviated the early apoptotic level of AC16 cell after hypoxia treatment (Figures 7G,H).

\section{DISCUSSION}

For the first time, our study applied microarray to identify the differences in circRNA expression levels between AMI patients and mild coronary artery stenosis patients. Results of microarray analysis were validated by RT-qPCR in larger samples (30 AMI patients, 30 mild coronary artery stenosis patients, and 30 normal coronary artery volunteers) and in human cardiomyocytes AC16, which implied that the expression of circRNA_104761 was an effective biomarker for AMI diagnosis. Given that circRNAs and miRNAs interact each other, circRNAs may be involved in the biological process of AMI through sponge miRNAs (Tang et al., 2018; Faiza et al., 2019; Chowdhury et al., 2020; Khan et al., 2020). The target microRNAs of circRNA_104761 were predicted by TargetScan, miRanda, and circRNA-microRNA-mRNA network, and we found that circRNA_104761 could sponge microRNA-449 and microRNA34a. It is worth noting that miRNA-449 and miRNA-34a are closely linked to AMI.

circRNA_ 104761 may promote cardiomyocyte apoptosis through sponging miR-449. In the study of Zhang et al. (2019), they found that knocking down lncRNAX inactivation specific transcript (XIST) in the AMI rat model could downregulate the level of miRNA-449 and inhibit rat cardiomyocyte apoptosis, suggesting that miRNA-449 is directly involved in the regulation of MI. MiRNA-449 regulate gene expression posttranscriptionally through mRNA degradation or translational repression (Esquela-Kerscher and Slack, 2006). MiRNA-449 is down-regulated in various cancers and is a strong inducer of cell cycle arrest (including senescence) and apoptosis in tumor cell lines (Bou Kheir et al., 2011). MiRNA-449 regulates various pathways (Lize et al., 2011), including Notch (Marcet et al., 2011), p53, E2F1 (Lize et al., 2010; Noonan et al., 2010),Wnt (Iliopoulos et al., 2009), and cell cycle (Bou Kheir et al., 2011). Among them, miRNA-449 provides negative feedback on E2F pathway and positive feedback on the p53 pathway, strengthening E2F1-p53 interdependence (Lize et al., 2010). In response to DNA damage, 
the transcription p53 and E2F1 deregulated in cancer, and then they were activated to induce pro-apoptotic genes, which directly promote apoptosis (Lize et al., 2011). circRNA_104761 is downregulated in AMI, which may reduce sponge miRNA-449 and change p53 and E2F-1 pathways,increasing myocardial apoptosis.

Furthermore, circRNA_ 104761 may promote cardiomyocyte apoptosis through sponging miR-34a. Fan et al. (2013) observed that miRNA-34a promoted cardiomyocyte apoptosis by negatively regulating aldehyde dehydrogenase 2 (ALDH2), which was increased in circulation under myocardial infarction (MI) conditions. In addition, the miR-34a can act as p53-responsive genes, which can induce apoptosis and cell cycle arrest in tumor cell lines (Rockenfeller et al., 2010). MiRNA-34a regulates many target proteins, which induce cell apoptosis in p53-dependent manner, including bcl-2 (Cole et al., 2008), YY1 (Chen et al., 2011), Notch (Li et al., 2009), MAPK (Tivnan et al., 2011), and DLL1 (Lewis et al., 2003), or independent manner. In AMI, the expression of circRNA_104761 is down-regulated, which may reduce the sponge function of circRNA_104761 on miRNA$34 \mathrm{a}$, resulting in changes in the p53-miRNA-34a axis, causing myocardial apoptosis.

There are several limitations in our study, which cannot be ignored. First, the number of subjects is not large enough, limiting the clinical value of circRNA_104761 as a potential biomarker. Also, a more diverse control group is needed, such as patients with moderate coronary artery stenosis and patients with severe coronary artery stenosis. Second, to further illustrate the application value of circRNA_104761 in AMI, animal models with knockdown or overexpression of circRNA_104761 are needed, and this experiment only carried out cell verification. In addition, due to limited funding, we only speculated the mechanism of circRNA_104761 via miRNA-449 and miRNA-34a to cause AMI by functional analysis. The relationship between circRNA_104761 and miRNA-499/miRNA-34a needs further investigation and verification. Finally, restricting the population to Asian males limited the generalizability of the findings to females and other races.

In summary, our results demonstrated that circRNA_104761 could not only be an effective biomarker for AMI diagnosis, but also differentiate normal coronary artery, mild coronary artery stenosis,and AMI. This study also identified that knockdown of circRNA_104761 with siRNA aggravated hypoxiainduced cardiomyocytes injury in $\mathrm{AC} 16$, and overexpression of circRNA_104761 alleviated hypoxia-induced injury.

\section{REFERENCES}

Anderson, J. L., and Morrow, D. A. (2017). Acute myocardial infarction. N. Engl. J. Med. 376, 2053-2064.

Begum, S., Yiu, A., Stebbing, J., and Castellano, L. (2018). Novel tumour suppressive protein encoded by circular RNA, circ-SHPRH, in glioblastomas. Oncogene 37, 4055-4057. doi: 10.1038/s41388-018-0230-3

Bou Kheir, T., Futoma-Kazmierczak, E., Jacobsen, A., Krogh, A., Bardram, L., Hother, C., et al. (2011). miR-449 inhibits cell proliferation and is downregulated in gastric cancer. Mol. Cancer 10:29. doi: 10.1186/1476-4598-1029
Therefore, circRNA_104761 may be considered a potential therapeutic target.

\section{DATA AVAILABILITY STATEMENT}

The original contributions presented in the study are included in the article/Supplementary Material, further inquiries can be directed to the corresponding author.

\section{ETHICS STATEMENT}

The studies involving human participants were reviewed and approved by the Harbin Medical University Ethics Committee. The patients/participants provided their written informed consent to participate in this study.

\section{AUTHOR CONTRIBUTIONS}

WY, LS, XC, LL, XZ, and WL conceived, designed the study, and revised the manuscript. WY, JL, TL, LZ, and GL collected samples. WY, HZ, CZ, and YZ performed the experiments and analyzed the data. All authors approved the final version of the manuscript.

\section{FUNDING}

This work was supported by Heilongjiang Research Projects of Basic Scientific Research (No. 2018KYYWF-0492).

\section{ACKNOWLEDGMENTS}

The authors would like to express their gratitude to EditSprings (https://www.editsprings.com/) for the expert linguistic services provided.

\section{SUPPLEMENTARY MATERIAL}

The Supplementary Material for this article can be found online at: https://www.frontiersin.org/articles/10.3389/fgene. 2021.686116/full\#supplementary-material 
Cocquerelle, C., Mascrez, B., Hetuin, D., and Bailleul, B. (1993). Mis-splicing yields circular RNA molecules. FASEB J. 7, 155-160. doi: 10.1096/fasebj.7.1.7678559

Cole, K. A., Attiyeh, E. F., Mosse, Y. P., Laquaglia, M. J., Diskin, S. J., Brodeur, G. M., et al. (2008). A functional screen identifies miR-34a as a candidate neuroblastoma tumor suppressor gene. Mol. Cancer Res. 6, 735-742. doi: 10.1158/1541-7786.mcr-07-2102

Dai, Y., Yang, J., Gao, Z., Xu, H., Sun, Y., Wu, Y., et al. (2017). Atrial fibrillation in patients hospitalized with acute myocardial infarction: analysis of the china acute myocardial infarction (CAMI) registry. BMC Cardiovasc. Disord. 17:2. doi: 10.1186/s12872-016-0442-9

Du, W. W., Yang, W., Liu, E., Yang, Z., Dhaliwal, P., and Yang, B. B. (2016). Foxo3 circular RNA retards cell cycle progression via forming ternary complexes with p21 and CDK2. Nucleic Acids Res. 44, 2846-2858. doi: 10.1093/nar/gkw027

Esquela-Kerscher, A., and Slack, F. J. (2006). Oncomirs - microRNAs with a role in cancer. Nat. Rev. Cancer 6, 259-269. doi: 10.1038/nrc1840

Faiza, M., Tanveer, K., Fatihi, S., Wang, Y., and Raza, K. (2019). Comprehensive overview and assessment of microRNA target prediction tools in Homo sapiens and Drosophila melanogaster. Curr. Bioinform. 14, 432-445. doi: 10.2174/ 1574893614666190103101033

Fan, F., Sun, A., Zhao, H., Liu, X., Zhang, W., Jin, X., et al. (2013). MicroRNA34 a promotes cardiomyocyte apoptosis post myocardial infarction through down-regulating aldehyde dehydrogenase 2. Curr. Pharm. Des. 19, 4865-4873. doi: 10.2174/13816128113199990325

Gao, R., Patel, A., Gao, W., Hu, D., Huang, D., Kong, L., et al. (2008). Prospective observational study of acute coronary syndromes in China: practice patterns and outcomes. Heart 94, 554-560. doi: 10.1136/hrt.2007.1 19750

Geng, H. H., Li, R., Su, Y. M., Xiao, J., Pan, M., Cai, X. X., et al. (2016). The circular RNA Cdrlas promotes myocardial infarction by mediating the regulation of miR-7a on its target genes expression. PLoS One 11:e0151753. doi: 10.1371/ journal.pone.0151753

Gu, Y., Ke, G., Wang, L., Zhou, E., Zhu, K., and Wei, Y. (2017). Altered expression profile of circular RNAs in the serum of patients with diabetic retinopathy revealed by microarray. Ophthalm. Res. 58, 176-184. doi: 10.1159/000 479156

Hajar, R. (2016). Evolution of myocardial infarction and its biomarkers: a historical perspective. Heart Views 17, 167-172. doi: 10.4103/1995-705x.2 01786

Hansen, T. B., Jensen, T. I., Clausen, B. H., Bramsen, J. B., Finsen, B., Damgaard, C. K., et al. (2013). Natural RNA circles function as efficient microRNA sponges. Nature 495, 384-388. doi: 10.1038/nature11993

Iliopoulos, D., Bimpaki, E. I., Nesterova, M., and Stratakis, C. A. (2009). MicroRNA signature of primary pigmented nodular adrenocortical disease: clinical correlations and regulation of Wnt signaling. Cancer Res. 69, 32783282. doi: 10.1158/0008-5472.can-09-0155

Jeong, J. H., Seo, Y. H., Ahn, J. Y., Kim, K. H., Seo, J. Y., Chun, K. Y., et al. (2020). Performance of copeptin for early diagnosis of acute myocardial infarction in an emergency department setting. Ann. Lab. Med. 40, 7-14. doi: 10.3343/alm. 2020.40.1.7

Jiang, X.-W., Liu, Y., Huang, T.-S., and Zhu, X.-Y. (2019). MGB block ARMS Realtime PCR for diagnosis of CYP2C19 mutation in a chinese population. Curr. Bioinform. 14, 391-399. doi: 10.2174/15748936146661901091 54252

Khan, A., Zahra, A., Mumtaz, S., Fatmi, M. Q., and Khan, M. J. (2020). Integrated In-silico analysis to study the role of microRNAs in the detection of chronic kidney diseases. Curr. Bioinform. 15, 144-154. doi: 10.2174/ 1574893614666190923115032

Lewis, B. P., Shih, I. H., Jones-Rhoades, M. W., Bartel, D. P., and Burge, C. B. (2003). Prediction of mammalian MicroRNA targets. Cell 115, 787-798. doi: 10.1016/s0092-8674(03)01018-3

Li, Y., Guessous, F., Zhang, Y., Dipierro, C., Kefas, B., Johnson, E., et al. (2009). MicroRNA-34a inhibits glioblastoma growth by targeting multiple oncogenes. Cancer Res. 69, 7569-7576. doi: 10.1158/0008-5472.can-09 $-0529$

Liu, W., Jiang, X., Liu, Y., and Ma, Q. (2019). Bioinformatics analysis of quantitative PCR and reverse transcription PCR in detecting HCV
RNA. Curr. Bioinform. 14, 400-405. doi: 10.2174/1574893613666180703 103328

Lize, M., Klimke, A., and Dobbelstein, M. (2011). MicroRNA-449 in cell fate determination. Cell Cycle 10, 2874-2882. doi: 10.4161/cc.10.17. 17181

Lize, M., Pilarski, S., and Dobbelstein, M. (2010). E2F1-inducible microRNA $449 \mathrm{a} / \mathrm{b}$ suppresses cell proliferation and promotes apoptosis. Cell Death Differ. 17, 452-458. doi: 10.1038/cdd.2009.188

Lu, Y., Deng, X., Xiao, G., Zheng, X., Ma, L., and Huang, W. (2019). circ_0001730 promotes proliferation and invasion via the miR-326/Wnt7B axis in glioma cells. Epigenomics 11, 1335-1352. doi: 10.2217/epi-2019-0121

Luo, Q., Zeng, L., Zeng, L., Rao, J., Zhang, L., Guo, Y., et al. (2020). Expression and clinical significance of circular RNAs hsa_circ_0000175 and hsa_circ_0008410 in peripheral blood mononuclear cells from patients with rheumatoid arthritis. Int. J. Mol. Med. 45, 1203-1212.

Marcet, B., Chevalier, B., Luxardi, G., Coraux, C., Zaragosi, L. E., Cibois, M., et al. (2011). Control of vertebrate multiciliogenesis by miR-449 through direct repression of the Delta/Notch pathway. Nat. Cell Biol. 13, 693-699. doi: 10. $1038 /$ ncb2241

Noonan, E. J., Place, R. F., Basak, S., Pookot, D., and Li, L. C. (2010). miR-449a causes Rb-dependent cell cycle arrest and senescence in prostate cancer cells. Oncotarget 1, 349-358. doi: 10.18632/oncotarget.167

Rockenfeller, P., Ring, J., Muschett, V., Beranekt, A., Büttner, S., Gutierrez, D., et al. (2010). Fatty acids trigger mitochondrion-dependent necrosis. Cell Cycle 9, 2908-2914. doi: 10.4161/cc.9.14.12346

Roger, V. L., Go, A. S., Lloyd-Jones, D. M., Benjamin, E. J., Berry, J. D., Borden, W. B., et al. (2012). Executive summary: heart disease and stroke statistics-2012 update: a report from the American heart association. Circulation 125, 188-197.

Rybak-Wolf, A., Stottmeister, C., Glazar, P., Jens, M., Pino, N., Giusti, S., et al. (2015). Circular RNAs in the mammalian brain are highly abundant, conserved, and dynamically expressed. Mol. Cell 58, 870-885. doi: 10.1016/j.molcel.2015. 03.027

Tang, W., Wan, S., Yang, Z., Teschendorff, A. E., and Zou, Q. (2018). Tumor origin detection with tissue-specific miRNA and DNA methylation markers. Bioinformatics 34, 398-406. doi: 10.1093/bioinformatics/btx622

Tivnan, A., Tracey, L., Buckley, P. G., Alcock, L. C., Davidoff, A. M., and Stallings, R. L. (2011). MicroRNA-34a is a potent tumor suppressor molecule in vivo in neuroblastoma. BMC Cancer 11:33. doi: 10.1186/14712407-11-33

Wang, G., Qi, K., Zhao, Y., Li, Y., Juan, L., Teng, M., et al. (2013). Identification of regulatory regions of bidirectional genes in cervical cancer. BMC Med. Genom. 6(Suppl. 1):S5. doi: 10.1186/1755-8794-6-S1-S5

Wang, K., Long, B., Liu, F., Wang, J. X., Liu, C. Y., Zhao, B., et al. (2016). A circular RNA protects the heart from pathological hypertrophy and heart failure by targeting miR-223. Eur. Heart J. 37, 2602-2611. doi: 10.1093/eurheartj/ehv713

Wei, L., Liao, M., Gao, Y., Ji, R., He, Z., and Zou, Q. (2014). Improved and promising identification of human MicroRNAs by incorporating a high-quality negative set. IEEE ACM Trans. Comput. Biol. Bioinform. 11, 192-201. doi: $10.1109 / \mathrm{tcbb} .2013 .146$

Wei, L., Tang, J., and Zou, Q. (2017). Local-DPP: an improved DNA-binding protein prediction method by exploring local evolutionary information. Inform. Sci. 384, 135-144. doi: 10.1016/j.ins.2016.06.026

Xu, Z., Tie, X., Li, N., Yi, Z., Shen, F., and Zhang, Y. (2020). Circular RNA hsa_circ_0000654 promotes esophageal squamous cell carcinoma progression by regulating the miR-149-5p/IL-6/STAT3 pathway. IUBMB Life 72, 426-439. doi: 10.1002/iub.2202

Yang, H., Luo, Y., Ren, X., Wu, M., He, X., Peng, B., et al. (2021). Risk prediction of diabetes: big data mining with fusion of multifarious physical examination indicators. Inform. Fusion [Epub ahead of print]. doi: 10.1016/j.inffus.2021.02. 015

Yang, Y. H., Ma, C., Wang, J. S., Yang, H., Ding, H., Han, S. G., et al. (2020). Prediction of N7-methylguanosine sites in human RNA based on optimal sequence features. Genomics 112, 4342-4347. doi: 10.1016/j.ygeno.2020.07.035

Zeng, X., Lin, W., Guo, M., and Zou, Q. (2017). A comprehensive overview and evaluation of circular RNA detection tools. PLoS Computat. Biol. 13:e1005420. doi: 10.1371/journal.pcbi. 1005420 
Zeng, X., Zhong, Y., Lin, W., and Zou, Q. (2020). Predicting disease-associated circular RNAs using deep forests combined with positive-unlabeled learning methods. Brief. Bioinform. 21, 1425-1436. doi: 10.1093/bib/bbz080

Zhai, Y., Chen, Y., Teng, Z., and Zhao, Y. (2020). Identifying antioxidant proteins by using amino acid composition and protein-protein interactions. Front. Cell Dev. Biol. 8:591487. doi: 10.3389/fcell.2020. 591487

Zhang, M., Liu, H. Y., Han, Y. L., Wang, L., Zhai, D. D., Ma, T., et al. (2019). Silence of lncRNA XIST represses myocardial cell apoptosis in rats with acute myocardial infarction through regulating miR-449. Eur. Rev. Med. Pharmacol. Sci. 23, 8566-8572.

Zhao, Y., Wang, F., Chen, S., Wan, J., and Wang, G. (2017). Methods of MicroRNA promoter prediction and transcription factor mediated regulatory network. Biomed. Res. Int. 2017:7049406.
Zhao, Y., Wang, F., and Juan, L. (2015). MicroRNA promoter identification in arabidopsis using multiple histone markers. Biomed. Res. Int. 2015:861402.

Conflict of Interest: The authors declare that the research was conducted in the absence of any commercial or financial relationships that could be construed as a potential conflict of interest.

Copyright (C) 2021 Yang, Sun, Cao, Li, Zhang, Li, Zhao, Zhan, Zang, Li, Zhang, Liu and $\mathrm{Li}$. This is an open-access article distributed under the terms of the Creative Commons Attribution License (CC BY). The use, distribution or reproduction in other forums is permitted, provided the original author(s) and the copyright owner(s) are credited and that the original publication in this journal is cited, in accordance with accepted academic practice. No use, distribution or reproduction is permitted which does not comply with these terms. 